\title{
Prevalence and Characteristics of Subjects with Obstructive Sleep Apnea among Adults with Insomnia Disorder
}

\author{
Jeewon Lee, MD, PhD, Ji Ho Choi, MD, PhD², Shin-Gyeom Kim, MD, PhD \\ 'Departments of Psychiatry, ${ }^{2}$ Otorhinolaryngology-Head and Neck Surgery, Soonchunhyang University College of Medicine, Bucheon Hospital, \\ Bucheon, Korea
}

\begin{abstract}
The aim of the present study was to evaluate the prevalence and characteristics of subjects with obstructive sleep apnea (OSA) among adults with insomnia disorder. Among 105 participants (mean age $=40.61 \pm 11.62$, male $=53.3 \%$ ) diagnosed with insomnia disorder who reported that they do not snore loudly, the prevalence of OSA was 35.2\%. Despite the high Apnea-Hypopnea Index, longer lighter stages of sleep, and more numbers of total arousal in the polysomnography, subjects with OSA had a lesser tendency to overestimate their sleep onset latency compared to those without OSA. This study highlights the importance of examining insomnia patients for OSA even when the patients do not report loud snoring. Future studies are needed to identify the specific clinical features of patients with co-existing OSA and insomnia.

Sleep Med Res 2019;10(2):108-112
\end{abstract}

\section{INTRODUCTION}

Complaints of insomnia is highly prevalent in the general population and in clinical practice [1]. Subjective report of difficulty initiating or maintaining sleep, occurring at least 3 times a week for 3 months, that causes functional impairment is enough for clinical diagnosis of insomnia disorder [2]. Routine polysomnography is not a prerequisite for the diagnosis of insomnia disorder. Still, investigating the presence of other sleep disorders such as obstructive sleep apnea (OSA) is very important. Hypnotics which are generally used for the treatment of insomnia should be used with caution for patients with OSA [3]. Also, OSA needs to be treated with a different modality such as continuous positive airway pressure (CPAP). Untreated OSA could have a negative impact on cardiovascular mortality [4].

OSA is underdiagnosed in general medical practice [5]. Clinical features of OSA are mainly loud snoring, poor sleep, daytime sleepiness or fatigue, and cessation of breathing during sleep observed by others. Poor sleep and daytime sleepiness or fatigue are also main symptoms of insomnia patients. Due to the overlapping symptoms of insomnia and OSA, it is difficult to suspect the presence of OSA in insomnia patients especially when there is no one to give information about one's snoring and cessation of breathing during sleep. Most clinicians consider the possibility of OSA only when the patient report disruptive, loud snoring [6].

The aim of the present study was to examine the prevalence of OSA in adults with insomnia who reported that they do not snore loudly. Also, we aimed to evaluate the clinical characteristics of the subjects with OSA among adults with insomnia. 


\section{METHODS}

Data for this research were drawn from a clinical study on adults with insomnia disorder which was conducted from November 2017 to December 2018. Baseline measurements of the participants were analyzed in order to examine the prevalence and characteristics of subjects with OSA. This study was approved by the Institutional Review Board of the Soonchunhyang University Bucheon Hospital (SCHBC 2017-07-029-022).

\section{Participants}

The inclusion criteria of the participants were adults aged 18 years to 59 years who met the diagnostic criteria of insomnia disorder according to the Diagnostic and Statistical Manual 5 criteria [2]. Exclusion criteria included major psychiatric disorders (schizophrenia, major depressive disorder, bipolar disorder, substance use disorder), developmental disorders (intellectual disability and autism spectrum disorder), neurocognitive disorders (delirium and dementia), neurologic disorders (epilepsy and cerebrovascular disease), any ongoing severe medical condition, history of suicidal attempt, consumption of any kind of sleep medications during the past month, and currently a shift-worker. Participants who had been diagnosed with OSA, those with body mass index (BMI) $>30 \mathrm{~kg} / \mathrm{m}^{2}$ and those with loud snoring were also excluded.

Participants were recruited through advertisements. Advertisements stated that we were recruiting subjects for the doubleblind, randomized, placebo-controlled study which was for the assessment of the efficacy and safety of passionflower extracts in adults with insomnia. They specified that subjects who took sleep medications during the past month and those who had been diagnosed with OSA or has loud snoring could not be enrolled in the study. Advertisements also stated that all participants would undergo polysomnography. All advertisements were approved by the Institutional Review Board. Written informed consent was obtained from each subject prior to participation. In this study, 105 participants with a score above 5 on Pittsburgh Sleep Quality Index (PSQI) were included in the analysis.

\section{Measurements}

All participants completed questionnaires consisting of demographic information and subjective reports of their total sleep time (sTST) and sleep onset latency (sSOL) during the past month. Also, sleep scales including Insomnia Severity Index (ISI), PSQI, and Epworth Sleepiness Scale (ESS) were completed by the participants. Center for Epidemiologic Studies Depression Scale and Beck Anxiety Inventory were used to evaluate the participants' level of depression and anxiety, respectively. The height and weight were measured in order to calculate the BMI of the participants.

Diagnosis of OSA was established using overnight polysomnography. All participants were monitored in an supervised sleep laboratory from 10:30 pm to 06:30 am. Participants with ApneaHypopnea Index (AHI) larger than 5 was diagnosed of OSA: mild sleep apnea $(5 \leq \mathrm{AHI}<15)$, moderate sleep apnea $(15 \leq$ AHI $<30)$ and severe sleep apnea: (AHI $\geq 30)$. Objective sleep parameters including total sleep time (oTST), sleep efficiency (SE), sleep onset latency (OSOL), wake after sleep onset, and number of total arousal were also obtained by the polysomnography.

\section{Statistical Analysis}

Group differences on the demographic variables and clinical data were analyzed using independent $t$-tests and chi-square tests. Data are given as mean \pm standard deviation. $\mathrm{p}$ values $<0.05$ were considered statistically significant. All statistical analyses were performed using the SPSS 20.0 (IBM Corp., Armonk, NY, USA).

\section{RESULTS}

\section{Prevalence of OSA}

Among 105 participants, the prevalence of OSA was 35.2\%: $22(21.1 \%)$ had mild OSA, $9(8.6 \%)$ had moderate OSA and 6 (5.7\%) had severe OSA. According to the AHI, participants were divided into two groups: non-OSA group $(\mathrm{n}=68)$ and OSA group $(\mathrm{n}=37)$.

\section{Demographic and Clinical Characteristics of the Subjects}

Demographic and clinical characteristics of the OSA and non-OSA group are compared in Table 1. The OSA group was older and predominant with male compared to the non-OSA group which was younger and predominant with female. The BMI was higher in the OSA group. Subjective sleep scales including ISI, PSQI, ESS did not show any difference between the two groups. In case of ISI, not only the total score which reflects the overall sleep quality but also both the scores for difficulty initiating and maintaining sleep didn't show any difference between the two groups (data not shown). Polysomnography results showed that the percentage of $\mathrm{N} 1$ was significantly higher and that of N2 and N3 was significantly lower in the OSA group compared to the non-OSA group. The number of total arousal was significantly higher in the OSA group.

\section{Discrepancy between Subjective and Objective Sleep Parameters}

Subjective reports and objective results of TST did not show any difference between the two groups (Table 2). The mean difference between sTST and oTST in the participants was $81.65 \pm$ 97.41. The participants had a tendency to underestimate their TST. The mean difference between sTST and oTST in the two groups were not statistically significant.

While objective SOL didn't show any significant difference be- 
tween the two groups, subjective SOL was significantly shorter in the OSA group compared to the non-OSA group (Table 2). Consequently, the mean difference between sSOL and oSOL was significantly smaller in the OSA group. The mean difference between sSOL and oSOL in the participants was $41.28 \pm 45.01$. Participants had a tendency to overestimate their SOL and those

Table 1. Demographic and clinical characteristics of the participants $(n=105)$

\begin{tabular}{|c|c|c|c|c|}
\hline Variables & Total $(\mathrm{n}=105)$ & Non-OSA $(n=68)$ & $\operatorname{OSA}(\mathrm{n}=37)$ & $\mathrm{p}$-value \\
\hline Age, years & $40.61 \pm 11.62$ & $38.16 \pm 11.86$ & $45.11 \pm 9.80$ & $0.003^{* *}$ \\
\hline Male, $\mathrm{n}(\%)$ & $56(53.3)$ & $20(29.4)$ & $29(78.4)$ & $<0.001$ \\
\hline Education, years & $15.08 \pm 2.21$ & $15.08 \pm 2.11$ & $15.09 \pm 2.42$ & 0.856 \\
\hline BMI, $\mathrm{kg} / \mathrm{m}^{2}$ & $23.69 \pm 2.64$ & $22.98 \pm 2.54$ & $24.99 \pm 2.32$ & $<0.001$ \\
\hline \multicolumn{5}{|l|}{ Subjective reports } \\
\hline sTST, min & $316.69 \pm 70.57$ & $311.65 \pm 67.40$ & $325.95 \pm 76.14$ & 0.335 \\
\hline sSOL, min & $57.45 \pm 41.60$ & $65.29 \pm 46.57$ & $43.04 \pm 25.19$ & $0.026^{*}$ \\
\hline ISI & $14.47 \pm 4.23$ & $14.74 \pm 4.07$ & $13.97 \pm 4.54$ & 0.597 \\
\hline PSQI & $8.64 \pm 1.49$ & $8.79 \pm 1.51$ & $8.35 \pm 1.42$ & 0.187 \\
\hline KESS & $9.25 \pm 4.27$ & $9.52 \pm 4.38$ & $8.76 \pm 4.09$ & 0.366 \\
\hline CES-D & $12.47 \pm 9.59$ & $13.24 \pm 10.13$ & $11.05 \pm 8.45$ & 0.333 \\
\hline BAI & $9.78 \pm 8.31$ & $9.97 \pm 8.11$ & $9.43 \pm 8.78$ & 0.340 \\
\hline \multicolumn{5}{|l|}{ Polysomnography } \\
\hline oTST, min & $398.33 \pm 62.13$ & $400.33 \pm 64.56$ & $394.66 \pm 61.13$ & 0.469 \\
\hline SE, \% & $83.18 \pm 13.35$ & $83.72 \pm 13.73$ & $82.19 \pm 12.74$ & 0.397 \\
\hline oSOL, min & $16.17 \pm 24.51$ & $16.19 \pm 21.50$ & $16.13 \pm 29.58$ & 0.258 \\
\hline Stage R latency, min & $119.43 \pm 69.45$ & $118.72 \pm 60.48$ & $120.73 \pm 84.394$ & 0.685 \\
\hline $\mathrm{N} 1, \%$ & $13.59 \pm 9.65$ & $10.38 \pm 5.60$ & $19.48 \pm 12.48$ & $<0.001$ \\
\hline $\mathrm{N} 2, \%$ & $63.54 \pm 9.17$ & $65.14 \pm 8.72$ & $60.60 \pm 9.36$ & $0.020^{*}$ \\
\hline $\mathrm{N} 3, \%$ & $4.20 \pm 5.99$ & $5.77 \pm 6.60$ & $1.31 \pm 3.06$ & $<0.001$ \\
\hline NREM, \% & $81.33 \pm 5.88$ & $81.29 \pm 5.48$ & $81.41 \pm 6.63$ & 0.888 \\
\hline REM, \% & $18.67 \pm 5.88$ & $18.71 \pm 5.48$ & $18.60 \pm 6.64$ & 0.891 \\
\hline WASO, min & $64.62 \pm 57.67$ & $62.03 \pm 62.04$ & $69.38 \pm 49.07$ & 0.185 \\
\hline Total arousal, n & $118.85 \pm 68.87$ & $93.84 \pm 45.21$ & $164.81 \pm 80.91$ & $<0.001$ \\
\hline AHI & $7.26 \pm 11.91$ & $1.52 \pm 1.19$ & $17.81 \pm 15.19$ & $<0.001$ \\
\hline
\end{tabular}

Data are presented as mean \pm standard deviation.

${ }^{*} \mathrm{p}<0.05,{ }^{* *} \mathrm{p}<0.01$.

OSA: obstructive sleep apnea, BMI: body mass index, sTST: subjective total sleep time, sSOL: subjective sleep onset lastency, ISI: Insomnia Severity Index, PSQI: Pittsburgh Sleep Quality Index, CES-D: Center for Epidemiologic Studies Depression Scale, BAI: Beck Anxiety Inventory, oTST: objective total sleep time, oSOL: objective sleep onset latency, SE: sleep efficiency, WASO: wake after sleep onset, AHI: Apnea-Hypopnea Index, KESS: Korean version of Epworth Sleepiness Scale.

Table 2. Discrepancy between subjective and objective sleep parameters in the participants

\begin{tabular}{lcccc}
\hline & Total $(\mathrm{n}=105)$ & Non-OSA $(\mathrm{n}=68)$ & OSA $(\mathrm{n}=37)$ & $\mathrm{p}$-value \\
\hline sTST, min & $316.69 \pm 70.57$ & $311.65 \pm 67.40$ & $325.95 \pm 76.14$ & 0.335 \\
oTST, min & $398.33 \pm 62.13$ & $400.33 \pm 64.56$ & $394.66 \pm 61.13$ & 0.469 \\
$\Delta$ TST (sTST-oTST), min & $-81.65 \pm 97.41$ & $-88.68 \pm 95.26$ & $-68.72 \pm 101.27$ & 0.318 \\
sSOL, min & $57.45 \pm 41.60$ & $65.29 \pm 46.57$ & $43.04 \pm 25.19$ & $0.026^{*}$ \\
oSOL, min & $16.17 \pm 24.51$ & $16.19 \pm 21.50$ & $16.13 \pm 29.58$ & 0.258 \\
$\Delta$ SOL (sSOL-oSOL), min & $41.28 \pm 45.01$ & $49.10 \pm 47.77$ & $26.91 \pm 35.75$ & $0.008^{*}$ \\
\hline
\end{tabular}

Data are presented as mean \pm standard deviation.

${ }^{*} \mathrm{p}<0.05$.

OSA: obstructive sleep apnea, sTST: subjective total sleep time, oTST: objective total sleep time, sSOL: subjective sleep onset latency, oSOL: objective sleep onset latency. 
without OSA had significantly greater tendency to overestimate their SOL than those with OSA.

\section{DISCUSSION}

In this study, the prevalence of OSA in adults with insomnia was $35.2 \%$. This is higher than the known prevalence of OSA in the generation population which has been reported to be $27 \%$ in men and 16\% in women in Korean population [7]. Previous studies have reported that the prevalence of comorbid OSA and insomnia is $22 \%$ to $67 \%$ [8]. Subjects with OSA were older, predominant with male and more overweight than subjects without OSA. The results are concordant with the known risk factors of OSA which include older age, male, and obesity [9]. It is noteworthy that the participants of the present study were those who are considered relatively low risk for OSA. Subjects who reported that they snored loudly and older adults aged 60 or older were excluded from this study. Obese patients with BMI $>30 \mathrm{~kg} / \mathrm{m}^{2}$ were also excluded.

Snoring loudly is one of the most significant clinical presentations of OSA [10]. To our knowledge, the present study is the first study to evaluate the prevalence of OSA in patients with insomnia who reported that they do not snore loudly. It could be suggested that subjective report of snoring is not reliable and many patients may not be aware of their own snoring. Therefore, even when patients report that they do not snore loudly, OSA should not be ruled out.

Yet the clinical usefulness of screening insomnia patient with polysomnography should be given more consideration. Most of the subjects diagnosed with OSA in this study were mild to moderate rather than severe OSA. Despite the well-established benefits of CPAP treatment in patients with OSA, it has been reported that patients with mild to moderate OSA have poor compliance to CPAP treatment [11]. This could imply that 5.7\% of the subjects who had severe OSA in this study could be the only people who would practically benefit from the diagnosis of OSA. Further identification of specific clinical presentation of co-existing insomnia and sleep apnea is needed.

In this study, subjects with OSA had higher percentage of N1 sleep which is consistent with the previous studies on sleep architecture in patients with OSA [12]. It is known that patients with OSA have longer lighter stages of sleep compared to healthy controls [13]. Also, subjects with OSA had more numbers of total arousal compared to those without OSA in this study. Interestingly, despite the high AHI, longer lighter stages of sleep, and more numbers of total arousal, subjects with OSA did not have a stronger tendency to underestimate their sleep duration or sleep quality. Subjective reports of sleep parameters and sleep scales did not show any difference between the two groups.

In this study, polysomnographic results of SOL didn't show any difference between the OSA and non-OSA group. This is inconsistent with the previous studies that have reported that OSA patients have shorter sleep latency than healthy subjects [14]. This inconsistency may be due to the relatively mild severity of the OSA in the subjects in this study. Majority (59.5\%) of the subjects in the OSA group had mild OSA. Severe OSA patients have been reported to have shorter sleep latency than mild and moderate OSA patients [15].

It is known that insomnia patients have a tendency to exaggerate their subjective report of sleep problems [16]. Participants in this study also underestimated their sleep time and overestimated their SOL. Interestingly, subjects with OSA had a smaller tendency to overestimate their SOL compared to those without OSA. Previous studies have suggested that patients with OSA have less sleep misperception compared to patients with insomnia, even if they sleep less [17]. In a study that compared insomnia patients to insomnia patients with OSA, both groups did not show any difference regarding sleep perception of TST, which is consistent with our results [18]. No previous study have examined the discrepancy of subjective and objective report of SOL in patients with insomnia and those with both insomnia and OSA. In this study, the OSA group had a lesser tendency to exaggerate their SOL compare to the non-OSA group.

The present study has several limitations. First, only one night of polysomnography was conducted. Polysomnographic sleep parameters could not have accurately reflected the participants' usual sleep state. Second, participants were comprised of relatively mild to moderate severity of insomnia due to the many exclusion criteria. Generalizability of the findings to patients diagnosed with insomnia disorder with more severe symptoms may be limited. Third, participants were recruited through advertisements which had stated that participants would undergo a polysomnography and those with loud snoring can not be enrolled in the study. Since polysomnography is a relatively expensive test, subjects who wanted to undergo polysomnography could have given false information about their snoring to be enrolled in the study.

This study highlights the importance of examining insomnia patients for OSA even when the patients do not report loud snoring. The present study also extended the results of previous studies regarding characteristics of OSA and insomnia. Further identification of specific clinical presentation of co-existing insomnia and sleep apnea needs additional research. Future well-designed studies would be needed for better understanding of these coexisting sleep disorders.

\section{Acknowledgments}

This research was supported by High Value-added Food Technology Development Program, Ministry of Agriculture, Food and Rural Affairs, Republic of Korea (115044-03-3-HD030).

\section{Conflicts of Interest}

The authors have no financial conflicts of interest. 


\section{Authors' Contribution}

Conceptualization: Kim SG. Data curation: Lee J. Formal analysis: Choi JH. Funding acquisition: Kim SG. Investigation: Lee J. Supervision: Choi JH. Writing_original draft: Lee J. Writing_review \& editing: Kim SG.

\section{REFERENCES}

1. Simon GE, VonKorff M. Prevalence, burden, and treatment of insomnia in primary care. Am J Psychiatry 1997;154:1417-23.

2. American Psychiatry Association. Diagnostic and statistical manual of mental disorders, 5th edition: DSM-5. Arlington, VA: American Psychaitry Association;2013.

3. Mason M, Cates CJ, Smith I. Effects of opioid, hypnotic and sedating medications on sleep-disordered breathing in adults with obstructive sleep apnoea. Cochrane Database Syst Rev 2015:CD011090.

4. Martínez-García MA, Campos-Rodríguez F, Catalán-Serra P, SolerCataluña JJ, Almeida-Gonzalez C, De la Cruz Morón I, et al. Cardiovascular mortality in obstructive sleep apnea in the elderly: role of long-term continuous positive airway pressure treatment: a prospective observational study. Am J Respir Crit Care Med 2012;186:909-16.

5. Kapur V, Strohl KP, Redline S, Iber C, O'Connor G, Nieto J. Underdiagnosis of sleep apnea syndrome in U.S. communities. Sleep Breath 2002;6:49-54.

6. Miller JN, Berger AM. Screening and assessment for obstructive sleep apnea in primary care. Sleep Med Rev 2016;29:41-51.

7. Kim J, In K, Kim J, You S, Kang K, Shim J, et al. Prevalence of sleepdisordered breathing in middle-aged Korean men and women. Am J Respir Crit Care Med 2004;170:1108-13.

8. Smith S, Sullivan K, Hopkins W, Douglas J. Frequency of insomnia report in patients with obstructive sleep apnoea hypopnea syndrome
(OSAHS). Sleep Med 2004;5:449-56.

9. Young T, Skatrud J, Peppard PE. Risk factors for obstructive sleep apnea in adults. JAMA 2004;291:2013-6.

10. Stradling JR, Crosby JH. Predictors and prevalence of obstructive sleep apnoea and snoring in 1001 middle aged men. Thorax 1991;46:85-90.

11. Yetkin O, Kunter E, Gunen H. CPAP compliance in patients with obstructive sleep apnea syndrome. Sleep Breath 2008;12:365-7.

12. Verma A, Radtke RA, VanLandingham KE, King JH, Husain AM. Slow wave sleep rebound and REM rebound following the first night of treatment with CPAP for sleep apnea: correlation with subjective improvement in sleep quality. Sleep Med 2001;2:215-23.

13. Redline S, Kirchner HL, Quan SF, Gottlieb DJ, Kapur V, Newman A. The effects of age, sex, ethnicity, and sleep-disordered breathing on sleep architecture. Arch Intern Med 2004;164:406-18.

14. Kass JE, Akers SM, Bartter TC, Pratter MR. Rapid-eye-movementspecific sleep-disordered breathing: a possible cause of excessive daytime sleepiness. Am J Respir Crit Care Med 1996;154:167-9.

15. Lim LL, Tham KW, Fook-Chong SM. Obstructive sleep apnoea in Singapore: polysomnography data from a tertiary sleep disorders unit. Ann Acad Med Singapore 2008;37:629-36.

16. Harvey AG, Tang NK. (Mis)perception of sleep in insomnia: a puzzle and a resolution. Psychol Bull 2012;138:77-101.

17. Pinto LR Jr, Pinto MC, Goulart LI, Truksinas E, Rossi MV, Morin CM, et al. Sleep perception in insomniacs, sleep-disordered breathing patients, and healthy volunteers--an important biologic parameter of sleep. Sleep Med 2009;10:865-8.

18. Choi SJ, Suh S, Ong J, Joo EY. Sleep misperception in chronic insomnia patients with obstructive sleep apnea syndrome: implications for clinical assessment. J Clin Sleep Med 2016;12:1517-25. 